pared with the published list of predicted iron lines in the sun's spectrum, with gratifying results.

In the regions covered by our observations, there are 23I solar lines in the red, identified by prediction as $F e$ I, and 268 in the violet - a total of 499. Of these predicted lines 28 per cent have now been observed in the arc. The percentages of observed lines, arranged by solar intensity, are distributed as follows:

Solar Int.
$\begin{gathered}\geqslant \\ \mathrm{I} \\ \mathrm{O} \\ -\mathrm{I} \\ -2 \\ -3\end{gathered}$

\begin{tabular}{ccc}
\multicolumn{3}{c}{ Percentage of Lines } \\
Red & Violet & Total \\
58 & 42 & 46 \\
55 & 22 & 34 \\
28 & 19 & 22 \\
30 & 13 & 23 \\
4 & 0 & 4
\end{tabular}

In addition, there are $\mathrm{I} 8$ unclassified iron lines in the red and $\mathrm{I}_{3} \mathrm{I}$ in the violet list that coincide with solar lines that have heretofore been wholly or partially unidentified. As soon as the laboratory measurements have been confirmed by additional observations with different gratings, doubtless many of these new lines will be classified, and definitively identified in the solar spectrum.

National Bureau of Standards, Washington, D. C.

\section{Kopal, Z. On the cause of light changes of Cepheid variables.}

It is pointed out that if the Cepheid variables are pulsating gas spheres characterized by no variation of pressure on the surface, the existence of the period-luminosity relation, combined with the assumption that the radiative properties of the Cepheids do not differ greatly from those of normal stars of the same spectra, is sufficient to define an empirical mass-radius-luminosity law which these variables should obey. The form of this law is, however, found to differ so drastically from any theoretical mass-radius-luminosity law to be expected for a wide variety of physically reasonable models that we seem, in effect, to be confronted with a choice between two alternatives: Either the Cepheid variables conforming to the period-luminosity relation do not constitute a homologous family of stars, in which case it is rather difficult to see why they should have otherwise so many properties in common; or the period of their light variation bears no relation to the frequency of a free radial oscillation with a node at the center and a loop on the surface.

Massachusetts Institute of Technology, Cambridge, Mass.
McClenahan, W.S. A new micrometer machine for measuring photographic films of meridian circle division marks.

A new instrument for measuring the photographic records of the meridian circle division marks was developed by members of the Positional Astronomy Division and constructed in the Dominion Observatory machine shop. The development was initiated by a similar method used at the Dominion Astrophysical Observatory at Victoria, B. C., for measuring stellar spectrograms.

The photographic record is projected on a plate which is controlled by weights and micrometer screws. Single lines on the plate are used to bisect the images. The pitch of the micrometer screw is $2 \mathrm{~mm}$. The head of the micrometer is graduated to Ioo parts and estimations made to thousandths. Magnification, approximately six, is such that one revolution of the screw is equal to $40^{\prime \prime}$.

Four microscopes are used with the meridian circle. A fine adjustment screw is provided to adjust the magnification for each film. A second screw of one $\mathrm{mm}$ pitch is used to reset the line on the division mark when the micrometer head is at zero.

Preliminary readings indicate that the settings can be easily made and the pointings show less variation than present microscope readings.

$$
\text { Dominion Observatory, }
$$
Ottawa, Canada.

\section{McLaughlin, Dean B. The spectrum of Nova Lacertae I950.}

This nova had the full complement of absorption systems:"pre-maximum," "principal," "diffuse enhanced," and "Orion." The hydrogen lines showed remarkable multiplicity. Spectral changes that accompanied fluctuations of light were strikingly similar to those of Nova Geminorum 19I2. Nitrogen "flaring" occurred long after the nebular stage was well advanced, in May and June.

Velocities of absorption components are tabulated in the table. Although the first observations were made after maximum light, the premaximum spectrum was still strong on January 27. By February I, it had weakened and the principal system was dominant. Oscillations of the Orion lines were correlated with changes of brightness of the star. Greatest velocities (numerically) occurred near minima of light, as in other novae. 